\title{
iPTF16abc and the population of Type Ia supernovae: comparing the photospheric, transitional, and nebular phases
}

\author{
S. Dhawan, ${ }^{1 \star}$ M. Bulla, ${ }^{1 \star}$ A. Goobar, ${ }^{1 \star}$ R. Lunnan, ${ }^{2}$ J. Johansson, ${ }^{3}$ C. Fransson, ${ }^{2}$ S. \\ R. Kulkarni, ${ }^{4}$ S. Papadogiannakis ${ }^{1}$ and A. A. Miller ${ }^{5,6}$ \\ ${ }^{1}$ The Oskar Klein Centre, Physics Department, Stockholm University, SE-106 91 Stockholm, Sweden \\ ${ }^{2}$ Department of Astronomy, The Oskar Klein Centre, Stockholm University, Alba Nova University Centre, SE-106 91 Stockholm, Sweden \\ ${ }^{3}$ Department of Physics and Astronomy, Division of Astronomy and Space Physics, Uppsala University, Box 516, SE 75120 Uppsala, Sweden \\ ${ }^{4}$ Division of Physics, Mathematics and Astronomy, California Institute of Technology, Pasadena, CA 91125, USA \\ ${ }^{5}$ Center for Interdisciplinary Exploration and Research in Astrophysics (CIERA) and Department of Physics and Astronomy, Northwestern University, 2145 \\ Sheridan Road, Evanston, IL 60208, USA \\ ${ }^{6}$ The Adler Planetarium, Chicago, IL 60605, USA
}

Accepted 2018 July 9. Received 2018 June 11; in original form 2018 February 2

\begin{abstract}
Key information about the progenitor system and the explosion mechanism of Type Ia supernovae (SNe Ia) can be obtained from early observations, within a few days from explosion. iPTF16abc was discovered as a young SN Ia with excellent early time data. Here, we present photometry and spectroscopy of the $\mathrm{SN}$ in the nebular phase. A comparison of the early time data with a sample of SNe Ia shows distinct features, differing from normal $\mathrm{SNe}$ Ia at early phases but similar to normal SNe Ia at a few weeks after maximum light (i.e. the transitional phase) and well into the nebular phase. The transparency time-scales $\left(t_{0}\right)$ for this sample of SNe Ia range between $\sim 25$ and $41 \mathrm{~d}$ indicating a diversity in the ejecta masses. $t_{0}$ also weakly correlates with the peak bolometric luminosity, consistent with the interpretation that $\mathrm{SNe}$ with higher ejecta masses would produce more ${ }^{56} \mathrm{Ni}$. Comparing the $t_{0}$ and the maximum luminosity, $L_{\max }$ distribution of a sample of $\mathrm{SNe}$ Ia to predictions from a wide range of explosion models we find an indication that the sub-Chandrasekhar mass models span the range of observed values. However, the bright end of the distribution can be better explained by Chandrasekhar mass delayed detonation models, hinting at multiple progenitor channels to explain the observed bolometric properties of SNe Ia. iPTF16abc appears to be consistent with the predictions from the $M_{\mathrm{ch}}$ models.
\end{abstract}

Key words: supernovae: general - supernova: individual (iPTF16abc).

\section{INTRODUCTION}

Type Ia supernovae (SNe Ia) have long been linked to the explosion of a C/O white dwarf (WD) in a binary system (Hoyle \& Fowler 1960), which has been confirmed by observed limits on the progenitor (Nugent et al. 2011; Bloom et al. 2012). However, there is still heated debate about the fundamental physical properties of the system, e.g. the mass of the progenitor, the nature of the companion, and the mechanism of the explosion (see Hillebrandt et al. 2013; Maoz, Mannucci \& Nelemans 2014, for a review). Many attempts to answer these open questions regarding the physics of $\mathrm{SNe}$ Ia

\footnotetext{
^E-mail: suhail.dhawan@fysik.su.se (SD); mattia.bulla@fysik.su.se (MB);
} ariel@fysik.su.se (AG) have concentrated on observations near maximum light. These observations have been critical to derive global parameters for $\mathrm{SNe}$ Ia, e.g. synthesized ${ }^{56}$ Nimass, total ejecta mass (Stritzinger et al. 2006; Scalzo et al. 2014; Dhawan et al. 2016, 2017). However, there is a wealth of information available in observations shortly after explosion as well as at late times ( $\sim$ a year after maximum, i.e. the nebular phase, e.g. Maguire et al. 2016; Graham et al. 2017). Early-time observations can shed light on the interaction between the SN ejecta and its companion (Kasen 2010), and can be used to constrain the size of the companion. The signature of such an interaction can be seen as a sharp excess in the ultraviolet (UV) and blue flux at early epochs (e.g. Cao et al. 2015), however, these pulses can be interpreted differently as in Kromer et al. (2016) and Noebauer et al. (2017). Very early-time observations of SNe Ia (Zheng 
et al. 2013, 2014; Goobar et al. 2014, 2015; Marion et al. 2016; Hosseinzadeh et al. 2017; Miller et al. 2018; Jiang et al. 2017) have shown a diversity in their behaviour shortly after explosion and are a rich source of information regarding the progenitor system and the explosion mechanism.

iPTF16abc was discovered shortly after explosion and showed some interesting early time features. It has a linear rise for the first $3 \mathrm{~d}$ after the time of first light, blue colours at early times compared to normal SNe (e.g. SN 2011 fe, Nugent et al. 2011), strong carbon features in early spectra that disappear after $\sim 7 \mathrm{~d}$ (Miller et al. 2018) and the near absence of the Si II $6355 \AA$ in the earliest spectrum. Here, we present a nebular spectrum of iPTF16abc and analyse the photospheric (i.e. pre-maximum), transitional (i.e. $\sim+30$ to $+100 \mathrm{~d}$ ), and nebular $(\sim+300 \mathrm{~d})$ phase properties of iPTF16abc in context of $\mathrm{SNe}$ in the literature.

The observations of the early, photospheric phase mostly probe the outer layers of the ejecta, the late phase, when the $\gamma$-ray escape fraction increases (Jeffery 1999; Stritzinger et al. 2006), is sensitive to the inner layers of the SN ejecta, which are dominated by iron group elements (IGEs). We, therefore, aim to answer if the features seen in the early phase also manifest at late epochs and hence, whether they are a result of only the composition of the outer layers of the ejecta or also due to the inner core.

The structure of the paper is as follows. We present the dataset in Section 2. We compare the properties of iPTF16abc to a sample of $\mathrm{SNe}$ in Section 3. We discuss our results in Section 4 and conclude in Section 5.

\section{DATA}

The intermediate Palomar Transient Factory (iPTF) reported the discovery of iPTF16abc (IAU name: SN2016bln), located 170 arcsec from the galaxy NGC 5221 at a redshift of $z=0.023$ and RA, Dec. $=13: 34: 45.492+13: 51: 14.30(\mathrm{~J} 2000)$. The line of sight that has low Milky Way reddening with $E(B-V)_{\mathrm{MW}}=0.028 \mathrm{mag}$ (Schlafly \& Finkbeiner 2011). The supernova was discovered on 2016 April 4.4 and the first detection was on 2016 April 3.4, the last non-detection was on 2016 April 2.4 (see Ferretti et al. 2017, for details). The distance to the host galaxy was calculated using a value of $H_{0}=70 \mathrm{kms}^{-1} \mathrm{Mpc}^{-1}$ and standard cosmology (i.e. flat, $\Omega_{\mathrm{m}}=0.3$ ) which corresponds to a distance of $100.24 \pm 4.3 \mathrm{Mpc}$ (using a peculiar velocity error on the redshift of $300 \mathrm{kms}^{-1}$ ), or a distance modulus of $35.00 \pm 0.10 \mathrm{mag}$. Note that since the $\mathrm{SN}$ is in the linear part of the Hubble diagram $(z=0.023)$, the effect of the assumed cosmology, apart from $H_{0}$ is very small.

As part of a late phase follow-up of iPTF16abc, we obtained a spectrum with the Low Resolution Imaging Spectrometer (LRIS) on the Keck telescope at $+342.4 \mathrm{~d}$. The spectrum was obtained as part of program C299 (PI: Kulkarni). It was reduced using the standard LRIS reduction pipeline, Ipipe, ${ }^{1}$ written in IDL . A summary of the spectra is provided in Table 1. Moreover, we also present photometry at similar epochs with DECam as part of the Dark Energy Camera Legacy Survey (DECaLS), reduced using standard point spread function fitting photometry routines in PYTHON.

In this study, we also analyse the data presented in Miller et al. (2018) and Ferretti et al. (2017). To this data, we add multiband data for a sample of SNe Ia from the literature. Since we analyse the multiband and bolometric properties of a number of SNe Ia, we require coverage from $u \rightarrow H$. We use data from the Carnegie

${ }^{1}$ http://www.astro.caltech.edu/dperley/programs/lpipe.html
Supernova Project (CSP; Contreras et al. 2010; Stritzinger et al. 2011) and the Center for Astrophysics (CfA) supernova program (Friedman et al. 2015) .

\section{RESULTS}

\subsection{Spectroscopy}

Fig. 1 shows observed spectra of iPTF16abc from -15.3 to +8.5 d relative to $B$-band maximum and Fig. 2 from +29.2 to $+342.4 \mathrm{~d}$, compared to those of some normal SNe Ia at similar epochs $( \pm 2.5$ d). Specifically, the comparison sample comprises spectra for the well-studied and nearby SN 2011fe (Nugent et al. 2011; Pereira et al. 2013; Maguire et al. 2014; Mazzali et al. 2014; Taubenberger et al. 2015) together with those for normal SNe Ia in the CSP sample of Table 2 that are characterized by $E(B-V)<0.2$ mag and no high-velocity features (i.e. SN2004ey, SN2005el, SN2005na, SN2006ax, and SN2008hv, Folatelli et al. 2013). We also plot the available spectra for the overluminous SN 1991T (Filippenko et al. 1992; Phillips et al. 1992; Ruiz-Lapuente et al. 1992; Gómez \& López 1998; Silverman, Ganeshalingam \& Filippenko 2013) and SN 1999aa (Garavini et al. 2004; Matheson et al. 2008; Silverman et al. 2013) in Figs 1 and 2.

Spectra of iPTF16abc before maximum show distinct peculiarities compared to the bulk of normal SNe Ia at these early epochs (Miller et al. 2018). The most striking difference is found across the $\mathrm{Si}$ II $\lambda 6355$ and $\mathrm{Ca}$ II near-infrared (NIR) triplet features around 6000 and $8000 \AA$, respectively. Both these features are usually strong in pre-maximum spectra of normal SNe Ia, while they are much weaker in iPTF16abc. An SNID classification of the $-11 \mathrm{~d}$ spectrum in Fig. 1 points to a best match with SN 1999aa, an overluminous, peculiar SN Ia (Garavini et al. 2004), which is consistent with the deep Ca H\&K feature in iPTF16abc that is not seen in SN 1991 T (Fig. 1). The early time (<-10 d) colours for iPTF16abc are significantly bluer (see Miller et al. 2018) and the colour evolution is significantly flatter than other overluminous $\mathrm{SNe}$ with such early observations (e.g. SN2012fr; Contreras et al. 2018). We emphasize, however, that our results are independent of the classification of iPTF16abc as a 91T-like, 99aa-like or normal SN Ia. As we will discuss in Section 4, this behaviour is consistent with the suggestion from Miller et al. (2018) that strong mixing could have occurred in the ejecta of iPTF16abc.

Despite the pronounced differences seen at early epochs, the spectral time evolution of iPTF16abc from peak brightness to about a year after is remarkably similar to the one observed in normal SNe Ia which have been shown to be similar to 91T-like/99aa-like SNe (Filippenko et al. 1992; Garavini et al. 2004), also consistent with the SNID classification from the post-maximum spectrum, presented in Miller et al. (2018). Good agreements with the comparison spectra are found at all epochs both in terms of colours (see also Miller et al. 2018) and velocities/strength of individual features. The similarities between iPTF16abc and the comparison sample extend up to transitional and nebular phases ( $\gtrsim 30 \mathrm{~d})$, when the inner regions of the $\mathrm{SN}$ ejecta are probed.

\subsubsection{Nebular phase}

Connections between SN Ia properties at early times and those at epochs $\gtrsim 150 \mathrm{~d}$ have been proposed, including correlations between the strength of the Fe $\lambda 4700$ feature and $\Delta m_{15, B}$ (Mazzali et al. 1998, but see also Blondin et al. 2012) and between nebular line 
Table 1. Log of spectroscopic observations.

\begin{tabular}{ccccc}
\hline Date & Phase (d) & Telescope + Instrument & Range $(\AA)^{\text {Reference }^{a}}$ \\
\hline $2016-04-05$ & -15.3 & Gemini-North+GMOS & $3800-9200$ & M18 \\
$2016-04-10$ & -10.9 & Keck-I+LRIS & $3055-10411$ & $3300-9998$ \\
$2016-04-13$ & -7.8 & LCO-2m+FLOYDS & $3300-9999$ & M18 \\
$2016-04-25$ & +3.7 & LCO-2m+FLOYDS & $3301-9999$ & M18 \\
$2016-04-30$ & +8.5 & LCO-2m+FLOYDS & $4000-8998$ & M18 \\
$2016-05-21$ & +29.2 & LCO-2m+FLOYDS & $4000-8998$ & M18 \\
$2016-06-03$ & +41.9 & LCO-2m+FLOYDS & $4001-8999$ & M18 \\
$2016-06-11$ & +49.7 & LCO-2m+FLOYDS & $3800-9300$ & M18 \\
$2016-06-23$ & +61.4 & LCO-2m+FLOYDS & & This work \\
$2017-03-29$ & +342.4 & &
\end{tabular}

Note: ${ }^{a}$ M18:Miller et al. (2018).
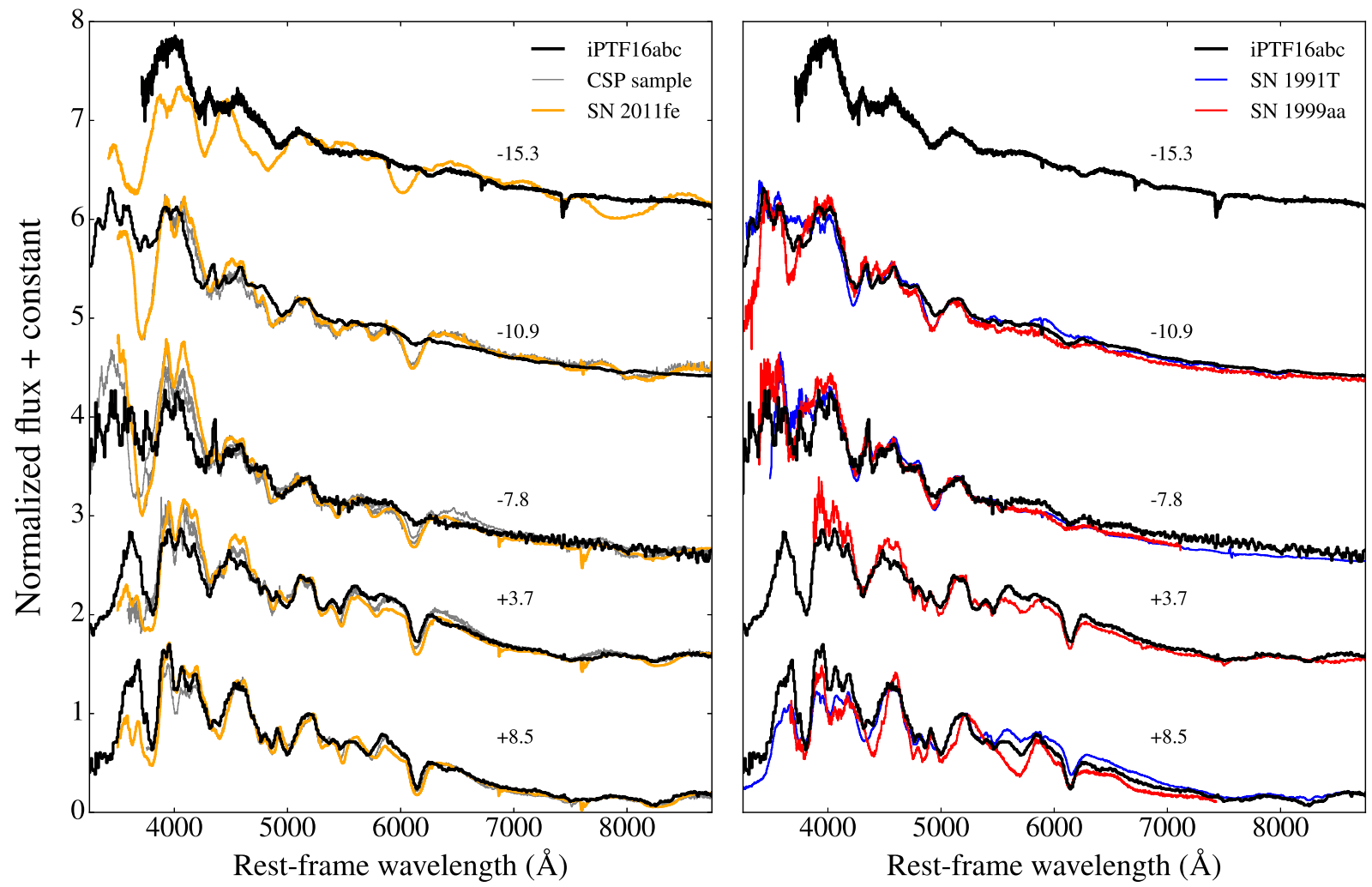

Figure 1. Left: the early time ( $<+10 \mathrm{~d}$ ) spectral time sequence of iPTF16abc (black) is compared to the spectra of SN 2011fe (orange), some normal SNe Ia in the CSP sample of Table 2 (grey). Right: the spectral time sequence is compared to SN 1991T and SN 1999aa at similar epochs ( \pm 2.5 d). Spectra in the left-hand panel are normalized by their values at $\sim 5200 \AA$, the top four in the right-hand panel by their values at $\sim 5500 \AA$ and the last spectra by their values at $\sim 5200 \AA$.

velocities and colours at peak (Maeda et al. 2011). Measuring latetime properties of iPTF16abc can improve our understanding of the observed peculiarities at early times. We obtained a spectrum of iPTF16abc at $+342.4 \mathrm{~d}$ close to a year after maximum light. In Fig. 2, we qualitatively compare the spectrum to SN 2011fe, SN 1991T, and SN 1999aa in the nebular phase and find no striking differences.

We measured the full width at half-maximum (FWHM) of the iron feature at $\sim 4700 \AA$ to be $17510 \pm 891 \mathrm{~km} \mathrm{~s}^{-1}$. Mazzali et al. (1998) found a relation between the FWHM of this feature 1.5and $\Delta m_{15}(B)$, although with a large sample of SNe, Blondin et al. (2012) find no strong relation. In Fig. 3, we plot the FWHM of the $4700 \AA$ feature for iPTF16abc with the sample of SNe from Blondin et al. (2012) and find that it is towards the higher end of the distribution of line widths but is consistent with the rest of the $\mathrm{SNe}$ in the literature.

\subsection{Bolometric properties}

Bolometric properties of SNe Ia have been demonstrated to hold important information regarding the underlying physical parameters of the progenitors, e.g. $M_{\mathrm{ej}},{ }^{56} \mathrm{Nimass}$ (Contardo, Leibundgut \& Vacca 2000; Stritzinger et al. 2006; Scalzo et al. 2014). We compute the bolometric light curves for $\mathrm{SNe}$ with photometry from $u$ to $H$ filters. The observed magnitudes are dereddened using the redden- 

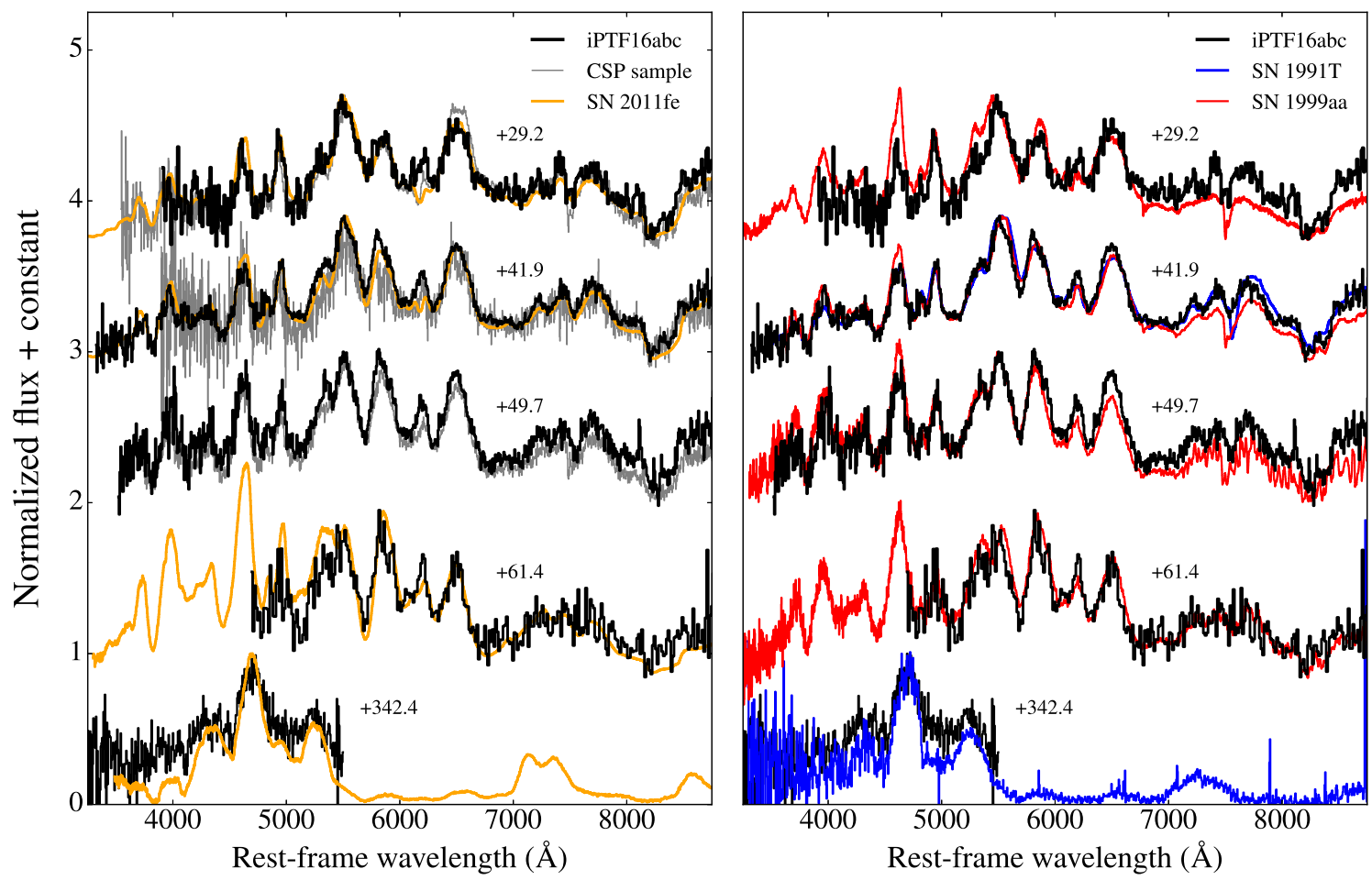

Figure 2. Same as Fig. 1, but for the transitional and the nebular phase. The top four spectra in the right-hand panel are normalized to their values at $\sim 5500$ $\AA$ and the last spectrum by its value at $\sim 4700 \AA$.

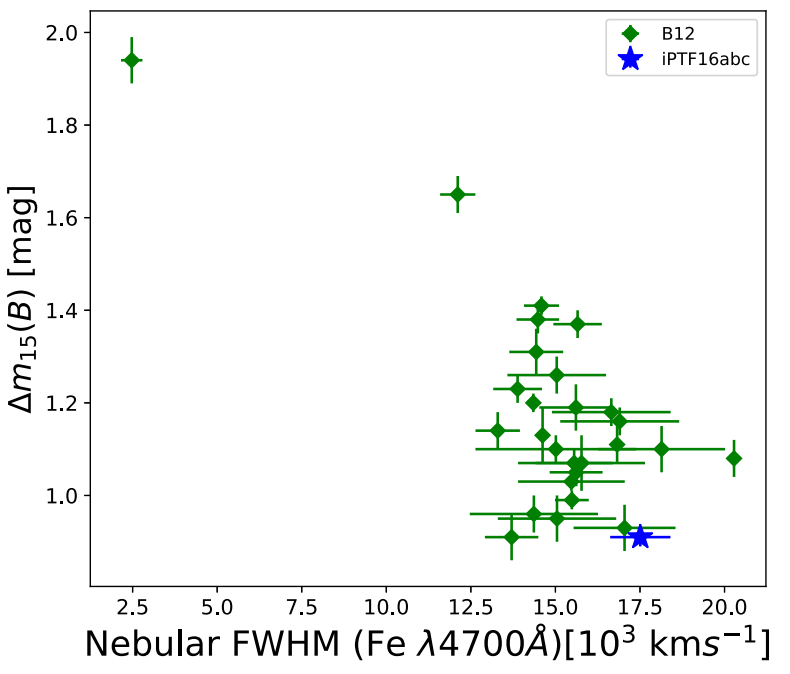

Figure 3. The $\Delta m_{15}(B)$ for the supernovae from the CfA SN program (Blondin et al. 2012) is plotted against the FWHM of the Fe $\lambda 4700 \AA$ A feature (green diamonds). iPTF16abc is plotted as the blue star and appears to be consistent with the distribution for the CfA sample.

ing law in Cardelli, Clayton \& Mathis (1989). Specifically, $E(B-$ $V$ ) and $R_{\mathrm{V}}$ values are computed using SNOOPY with the colour-stretch parameter (Burns et al. 2011, 2014) and are summarized in Table 2. Filter gaps and overlaps are treated as in Dhawan et al. $(2016,2017)$ and the apparent flux is converted to absolute fluxes using distances in Table 3. We use a cubic spline interpolation to derive the values of the maximum luminosity, $L_{\max }$, from the bolometric light curves. Here, we compare the early and late time bolometric properties of a sample of SNe Ia with the inferred values for iPTF16abc. Since
Table 2. This table contains the estimates for the decline rate in the $B$ band, $\Delta m_{15}$, host galaxy extinction, $E(B-V)_{\text {host }}$, and the total to selective absorption, $R_{\mathrm{V}}$, for the host galaxy of the $\mathrm{SN}$. The $\Delta m_{15}(B)$ is calculated with a Gaussian Process (GP) fit to the observed $B$-band data and the $E(B-$ $V)_{\text {host }}$ and $R_{\mathrm{V}}$ are calculated from SNOOPY fits (Burns et al. 2011) to multiband data.

\begin{tabular}{ccccc}
\hline SN & $\begin{array}{c}\Delta m_{15}(B) \\
(\mathrm{mag})\end{array}$ & $\begin{array}{c}E(B-V) \text { host } \\
(\mathrm{mag})\end{array}$ & $\begin{array}{c}\sigma \\
(\mathrm{mag})\end{array}$ & $R_{\mathrm{V}}$ \\
\hline SN2004eo & 1.42 & 0.128 & 0.024 & 0.9 \\
SN2004ey & 0.96 & 0.019 & 0.020 & 3.1 \\
SN2005el & 1.35 & 0.015 & 0.012 & 3.5 \\
SN2005ke & 1.78 & 0.263 & 0.033 & 0.8 \\
SN2005ki & 1.28 & 0.016 & 0.013 & 3.4 \\
SN2005M & 0.90 & 0.060 & 0.021 & 3.4 \\
SN2006ax & 1.00 & 0.016 & 0.015 & 2.9 \\
SN2006D & 1.43 & 0.134 & 0.025 & 2.5 \\
SN2006et & 0.90 & 0.254 & 0.025 & 1.9 \\
SN2006kf & 1.63 & 0.032 & 0.011 & 4.1 \\
SN2006mr & 1.83 & 0.089 & 0.039 & 2.9 \\
SN2007af & 1.17 & 0.178 & 0.024 & 2.1 \\
SN2007ax & 1.90 & 0.213 & 0.049 & 2.6 \\
SN2007bc & 1.59 & 0.207 & 0.025 & 1.8 \\
SN2007bd & 1.10 & 0.058 & 0.022 & 2.1 \\
SN2007le & 0.97 & 0.388 & 0.023 & 1.7 \\
SN2007on & 1.88 & 0.000 & 0.000 & 3.5 \\
SN2007S & 0.77 & 0.478 & 0.026 & 1.9 \\
SN2008bc & 0.85 & 0.000 & 0.000 & 3.1 \\
SN2008hv & 1.25 & 0.074 & 0.023 & 2.1 \\
SN2008ia & 1.29 & 0.066 & 0.016 & 3.8 \\
iPTF16abc & 0.91 & 0.070 & 0.016 & 3.1 \\
\hline
\end{tabular}

the light-curve shape for SNe Ia can depend on the host galaxy reddening for highly reddened SNe (Leibundgut 1988; Amanullah 
Table 3. Distances for the $\mathrm{SNe}$ in our sample. These were used to calculate the absolute (pseudo)-bolometric flux.

\begin{tabular}{ccc}
\hline SN & $\begin{array}{c}\mu \\
(\mathrm{mag})\end{array}$ & $\begin{array}{c}\sigma \\
(\mathrm{mag})\end{array}$ \\
\hline SN2004eo & 34.03 & 0.14 \\
SN2004ey & 34.01 & 0.15 \\
SN2005el & 34.05 & 0.14 \\
SN2005ke & 31.84 & 0.08 \\
SN2005ki & 34.74 & 0.11 \\
SN2005M & 35.0 & 0.09 \\
SN2006ax & 34.46 & 0.12 \\
SN2006D & 33.1 & 0.22 \\
SN2006et & 34.82 & 0.10 \\
SN2006kf & 34.78 & 0.10 \\
SN2006mr & 31.15 & 0.23 \\
SN2006X & 32.17 & 0.32 \\
SN2007af & 32.16 & 0.32 \\
SN2007ax & 32.2 & 0.27 \\
SN2007bc & 34.89 & 0.10 \\
SN2007bd & 35.73 & 0.07 \\
SN2007le & 31.88 & 0.36 \\
SN2007on & 31.45 & 0.08 \\
SN2007S & 34.07 & 0.14 \\
SN2008bc & 34.17 & 0.14 \\
SN2008fp & 32.16 & 0.32 \\
SN2008hv & 33.85 & 0.16 \\
SN2008ia & 34.97 & 0.10 \\
iPTF16abc & 35.01 & 0.09 \\
\hline
\end{tabular}

Table 4. The NIR second maximum timing for iPTF16abc.

\begin{tabular}{cc}
\hline Filter & $t_{2}$ \\
& $(\mathrm{~d})$ \\
\hline$Y$ & $34.4 \pm 1.23$ \\
$J$ & $34.2 \pm 1.23$ \\
$H$ & $33.2 \pm 1.4$ \\
\hline
\end{tabular}

\& Goobar 2011; Bulla et al. 2018), we only use SNe with $E(B-$ $V)<0.5$ mag. ${ }^{56} \mathrm{Ni}$ mass $\left(\mathrm{M}_{56 \mathrm{Ni}}\right)$ values are derived from $L_{\max }$ using Arnett's rule, i.e. assuming that the instantaneous rate of energy deposition equals the output flux at maximum (Arnett 1982):

$\epsilon_{\mathrm{Ni}}\left(t_{\mathrm{R}}, 1 \mathrm{M}_{\odot}\right)=\alpha \cdot\left(6.45 \cdot 10^{43} \mathrm{e}^{-t_{R} / 8.8}+1.45 \cdot 10^{43} \mathrm{e}^{-t_{\mathrm{R}} / 111.3}\right) \operatorname{erg~s}^{-1}(1)$

where $t_{\mathrm{R}}$ is the bolometric rise time and $\alpha$ is the parameter that accounts for deviations from Arnett's rule. These departures from $\alpha$ $=1$ could be due to the ${ }^{56} \mathrm{Ni}$ distribution, however, detailed model calculations of SNe Ia find that $\alpha=1$ with a scatter of $10-15$ per cent (Blondin et al. 2013, 2017). The impact of this on further parameter estimation is discussed below. Assuming Arnett's rule is obeyed and a rise time of $t_{\mathrm{R}}=19 \mathrm{~d}$ with an error of $3 \mathrm{~d}$ (Stritzinger et al. 2006; Dhawan et al. 2016), we can derive an $\mathrm{M}_{56_{\mathrm{Ni}}}$ from the computed value of $L_{\max }$ :

$$
\frac{\mathrm{M}_{56 \mathrm{Ni}}}{\mathrm{M}_{\odot}}=\frac{L_{\max }}{2.0( \pm 0.3) \times 10^{43} \mathrm{erg} \mathrm{s}^{-1}} .
$$

For iPTF16abc, the small difference in the inferred $\mathrm{M}_{56 \mathrm{Ni}}$ from Miller et al. (2018) is due to the different methods adopted for calculating the distances. We discuss the impact of the rise time in Section 4.1. We note that some SNe in our sample have also been studied in detail in the literature (e.g. SN2007on; Ashall et al. 2018). We find our inferred ${ }^{56}$ Nimass in good agreement with the values they report.
We also calculate the transparency time-scale $\left(t_{0}\right)$ for the sample of SNe, defined by Jeffery (1999) as a parameter that governs the time-varying $\gamma$-ray optical depth behaviour of a supernova. We determined $t_{0}$ by fitting the radioactive decay energy deposition to the late time (40-90 d) bolometric light curve:

$$
\begin{array}{r}
E_{\mathrm{dep}}=E_{\mathrm{Ni}}+E_{\mathrm{Coe}^{+}}+\left[1-\exp \left(-\tau_{\gamma}\right)\right] E_{\mathrm{Co} \gamma} \\
=\lambda_{\mathrm{Ni}} \mathrm{N}_{\mathrm{Ni} 0} \exp \left(-\lambda_{\mathrm{Ni}} \mathrm{t}\right) \mathrm{Q}_{\mathrm{Ni} \gamma} \\
+\lambda_{\mathrm{Co}} \mathrm{N}_{\mathrm{Ni} 0} \frac{\lambda_{\mathrm{Ni}}}{\lambda_{\mathrm{Ni}}-\lambda_{\mathrm{Co}}}\left[\exp \left(-\lambda_{\mathrm{Co}} \mathrm{t}\right)-\exp \left(-\lambda_{\mathrm{Ni}} \mathrm{t}\right)\right] \\
\times\left\{Q_{\mathrm{Coe}^{+}}+Q_{\mathrm{Co} \gamma}\left[1-\exp \left(-\tau_{\gamma}\right)\right]\right\},
\end{array}
$$

where the factor $\left(1-\exp \left(-\tau_{\gamma}\right)\right)$ is replaced by 1 for ${ }^{56} \mathrm{Ni}$ since complete trapping of $\gamma$-rays occurs at early times, when most of the light curve is powered by ${ }^{56} \mathrm{Ni}$, where $\lambda_{\mathrm{Ni}}$ and $\lambda_{\mathrm{Co}}$ are the e-folding decay times of 8.8 and $111.3 \mathrm{~d}$ for ${ }^{56} \mathrm{Niand}{ }^{56} \mathrm{Co}$, respectively. $Q_{\mathrm{Ni} \gamma}$ $(1.75 \mathrm{MeV})$ is the energy release per ${ }^{56} \mathrm{Ni} \rightarrow{ }^{56}$ Codecay. $Q_{\mathrm{Co} \gamma}(3.61$ $\mathrm{MeV})$ and $Q_{\mathrm{Co} e^{+}}(0.12 \mathrm{MeV})$ are the $\gamma$-ray and positron energies, respectively, released per ${ }^{56} \mathrm{Co} \rightarrow{ }^{56}$ Fedecay (see Stritzinger et al. 2006). Equation (3) is only applicable in the optically thin limit, when the thermalized photons can freely escape.

$\tau_{\gamma}$ is the mean optical depth, calculated by integrating from the point of emission to the surface of the ejecta (see Jeffery 1999, for a derivation of the expression). It has a simple $t^{-2}$ dependence, given as,

$\tau_{\gamma}=\frac{t_{0}^{2}}{t^{2}}$

where $t_{0}$ is the transparency time-scale, which by construction in Jeffery (1999) is the epoch at which the optical depth is unity.

The value of the transparency time-scale can be directly related to the total ejecta mass $\left(M_{\mathrm{ej}}\right)$ with the following equation (Jeffery 1999; Stritzinger et al. 2006; Dhawan et al. 2017)

$M_{\mathrm{ej}}=1.38 \cdot\left(\frac{1 / 3}{q}\right) \cdot\left(\frac{v_{\mathrm{e}}}{3000 \mathrm{kms}^{-1}}\right)^{2} \cdot\left(\frac{t_{0}}{36.80 \mathrm{~d}}\right)^{2} \mathrm{M}_{\odot}$.

Equation (5) encapsulates the capture rate of $\gamma$-rays in an expanding spherical volume for a given distribution of the radioactive source. We discuss the typical parameter values for canonical SN Ia models to map $t_{0}$ to $M_{\mathrm{ej}}$, however, we note that we do not use any calculations for $M_{\mathrm{ej}}$ in our analysis and only use the observed $t_{0}$ values. $q$ is a qualitative description of the distribution of the material within the ejecta, with one-third being a uniform distribution and higher values reflecting more centrally concentrated ${ }^{56} \mathrm{Ni}$ (more typical of subluminous 1991bg-like SNe Mazzali et al. 1997). The e-folding velocity $v_{\mathrm{e}}$ provides the scaling length for the expansion, which is $\sim 3000 \mathrm{~km} \mathrm{~s}^{-1}$ for Chandrasekhar-mass $\left(M_{\mathrm{ch}}\right)$ explosions corresponding to typical brightness $\mathrm{SNe}$ Ia and on average slightly lower for sub- $M_{\mathrm{ch}}$ WDs. For both $M_{\mathrm{ch}}$ and sub- $M_{\mathrm{ch}}$ models, it ranges between $\sim 2700-3200 \mathrm{~km} \mathrm{~s}^{-1}$. For an ejecta with $Y_{\mathrm{e}}=0.5$, which is appropriate for SNe Ia, the $\gamma$-ray opacity is a constant of $0.025 \mathrm{~cm}^{2}$ $\mathrm{g}^{-1}$ (Swartz, Sutherland \& Harkness 1995). Given the range of possible values for $q$ and $v_{\mathrm{e}}$, we do not proceed to infer $M_{\mathrm{ej}}$ for the $\mathrm{SNe}$ in our sample.

Because the UVOIR light curve is not truly bolometric, there is an implicit assumption that the flux redward of the $H$ band and bluewards of the $u$ band have very small contributions to the bolometric light curve. This assumption is supported by modelling that shows that the IR catastrophe does not occur until $\sim 1 \mathrm{yr}$ post-explosion or later, whereas typical values of $t_{0}$ are between 20 and $50 \mathrm{~d}$ and the line-blanketing opacity in the UV remains high (Blondin, Dessart \& Hillier 2015; Fransson \& Jerkstrand 2015). 
Table 5 Nebular phase photometry with DECam.

\begin{tabular}{lllll}
\hline MJD & Phase (d) & Filter & Magnitude & $\sigma$ \\
\hline & & & $(\mathrm{mag})$ & $(\mathrm{mag})$ \\
57818.30 & 320.20 & desg & 22.249 & 0.053 \\
57818.30 & 320.20 & desr & 23.323 & 0.053 \\
57814.39 & 316.29 & desi & 22.501 & 0.191 \\
\hline
\end{tabular}

Recent studies of the diagnostics of ejecta mass (e.g. Wilk et al. 2017) compute the $t_{+1 / 2}$ for different ejecta mass models. $t_{+1 / 2}$ is defined as the time the bolometric light curve takes to decline to half of its peak luminosity (Contardo et al. 2000). In Fig. 4, we plot the $t_{+1 / 2}$ against $t_{0}$ for the sample with sufficient data. Unsurprisingly, we find a strong correlation $(r \sim 0.85)$ for the objects in our sample, suggesting that the SNe that take longer to decline to half the peak luminosity also have optically thick ejecta for longer. Since Jeffery (1999) suggest that $t_{0}$ is directly related to $M_{\mathrm{ej}}$ and this equation has been used to calculate $M_{\mathrm{ej}}$ for SNe Ia (Stritzinger et al. 2006; Scalzo et al. 2014), this implies that $t_{+1 / 2}$ can also be used as a diagnostic for the ejecta mass.

\subsection{Near-infrared light curves}

The NIR morphology of SN Ia light curves has been shown to be markedly different from the optical (Elias et al. 1981, 1985). The SNe show a characteristic second peak in the $i z Y J H K$ filters (Mandel et al. 2009; Biscardi et al. 2012; Dhawan et al. 2015) and a less pronounced 'shoulder'-like feature in the $V$ and $r$ bands. Theoretical studies have shown that these features are caused by a recombination wave in the IGEs in the ejecta from doubly to singly ionized (Kasen 2006; Blondin et al. 2015), hence, the NIR second maximum is an important parameter to test whether the observed peculiarities in iPTF16abc could be arising due to the properties of the central IGE core. iPTF16abc shows a distinct second maximum in the $Y J H$ filters, consistent with the theoretical prediction for an SN Ia that produced $\sim 0.6 \mathrm{M}_{\odot}$ of ${ }^{56} \mathrm{Ni}$ (Kasen 2006 ).

The values for the timing of the second maximum $\left(t_{2}\right)$ for iPTF16abc are presented in Table 4 and shown in Fig. 5. The $t_{2}$ values of iPTF16abc are consistent within the range for normal SNe Ia (Biscardi et al. 2012; Dhawan et al. 2015), although they are at the higher end like 91T-like/99aa-like SNe. Dhawan et al. (2016) found a correlation between the peak (pseudo-) bolometric luminosity and $t_{2}$ for a sample of SNe with low reddening from the host galaxy dust. As shown in Fig. 5, the value for iPTF16abc lies on the expected relation.

\subsection{Nebular phase photometry}

iPTF16abc was also observed at epochs between +316 and $+320 \mathrm{~d}$ by the DECaLS survey in $g, r, z$ filters. The photometry on the DECaLS images is presented in Table 8. The decrease of 6.6 mag from peak to $\sim+300 \mathrm{~d}$ (see Fig. 6) is consistent with the expected value for normal SNe Ia (e.g. Stritzinger \& Sollerman 2007). We also find that the $r$-band absolute magnitude of $-11.4 \pm 0.11$ mag is within the range of observed values in the literature (Lair et al. 2006) at late phases of $\sim 1 \mathrm{yr}$ post-maximum. This further demonstrates that, at late epochs, the observed properties of iPTF16abc are within the observed distribution for normal SNe Ia. We note that since there is only $r$-band photometry available for SNe Ia at these late epochs (out of the three filters presented here), it is the only one for which we can compare iPTF16abc to literature values for a sample.

\section{DISCUSSION}

The early light curve and spectral evolution of iPTF16abc showed marked peculiarities that were interpreted as a signature of strong outward mixing of ${ }^{56} \mathrm{Ni}$ and/or interaction with diffuse material (Miller et al. 2018). In the case of strong mixing, the temperature and thus ionization level of the outer ejecta would be higher compared to the case of a centrally concentrated distribution of ${ }^{56} \mathrm{Ni}$ more commonly inferred for normal SNe Ia. This would explain why in iPTF16abc not only $\mathrm{C}_{\text {II }}$ features are stronger (Miller et al. 2018) but also $\mathrm{Si}$ II and $\mathrm{Ca}$ II features are weaker compared to what is seen for the bulk of normal SNe Ia. Shallow silicon and calcium features are also observed in peculiar events such as 2002cx-like and 1991T-like SNe Ia. Models for both 2002cx-like (pure deflagration models, e.g. Sahu et al. 2008; Kromer et al. 2013) and 1991T-like (Ruiz-Lapuente et al. 1992; Mazzali et al. 1995; Sasdelli et al. 2014; Fisher \& Jumper 2015; Seitenzahl et al. 2016; Zhang et al. 2016) $\mathrm{SNe}$ Ia invoke the presence of ${ }^{56} \mathrm{Ni}$ and IGEs in the outer ejecta, thus corroborating the idea that mixing might be responsible for the photometric and spectroscopic features seen in iPTF16abc. The nebular spectrum of iPTF16abc, however, looks consistent with the normal SN $2011 \mathrm{fe}$, and the measured line width of the $4700 \AA$ agrees with the width-decline rate relation for a sample of normal $\mathrm{SNe}$ from the literature (Fig. 3). The likely source of these peculiarities could therefore arise from the outer layers of the ejecta, and probably not from the central core of IGEs.

The transparency time-scale is strongly tied to the ejecta mass of the SN (Jeffery 1999; Stritzinger et al. 2006; Scalzo et al. 2014), therefore, given the uncertainties in driving the ejecta masses from $t_{0}$, we use $t_{0}$ directly for our analysis. As shown in Fig. 7, we find that the total $\mathrm{M}_{56 \mathrm{Ni}}$ (Table 5) is correlated with the transparency time-scale for our sample of SNe Ia (Table 6) such that objects with a longer $t_{0}$ produce more $\mathrm{M}_{56} \mathrm{Ni}_{\mathrm{i}}$ also indicating a possible positive relation of the ejecta mass with the amount of synthesized ${ }^{56}$ Niin agreement with previous studies (Stritzinger et al. 2006; Scalzo et al. 2014). iPTF16abc is consistent with this relation between $\mathrm{M}_{56 \mathrm{Ni}}$ and $t_{0}$. For both parameters, we assume a canonical rise time of $19 \mathrm{~d}$. In Section 4.1, we discuss the impact of this assumption.

The right-hand panel of Fig. 4 shows a weak correlation between the half-light time for the bolometric light curve $\left(t_{+1 / 2}\right)$ and the $\mathrm{M}_{56 \mathrm{Ni}}$ as in Contardo et al. (2000). Comparing the observed $t_{+1 / 2}$ from models for three different ejecta masses (Wilk et al. 2017), all producing $0.6 \mathrm{M}_{\odot}$ of $\mathrm{M}_{56 \mathrm{Ni}}$ (i.e. the same as the estimate for iPTF16abc Miller et al. 2018), we find that the range of $t_{+1 / 2}$ observed in SNe Ia is higher, possibly indicating that there is a large range of ejecta masses to explain the observations of the sample of SNe Ia. The observed $t_{+1 / 2}$ range could indicate that the sample of $\mathrm{SNe}$ corresponds to a range of ejecta masses. Since the lowest 

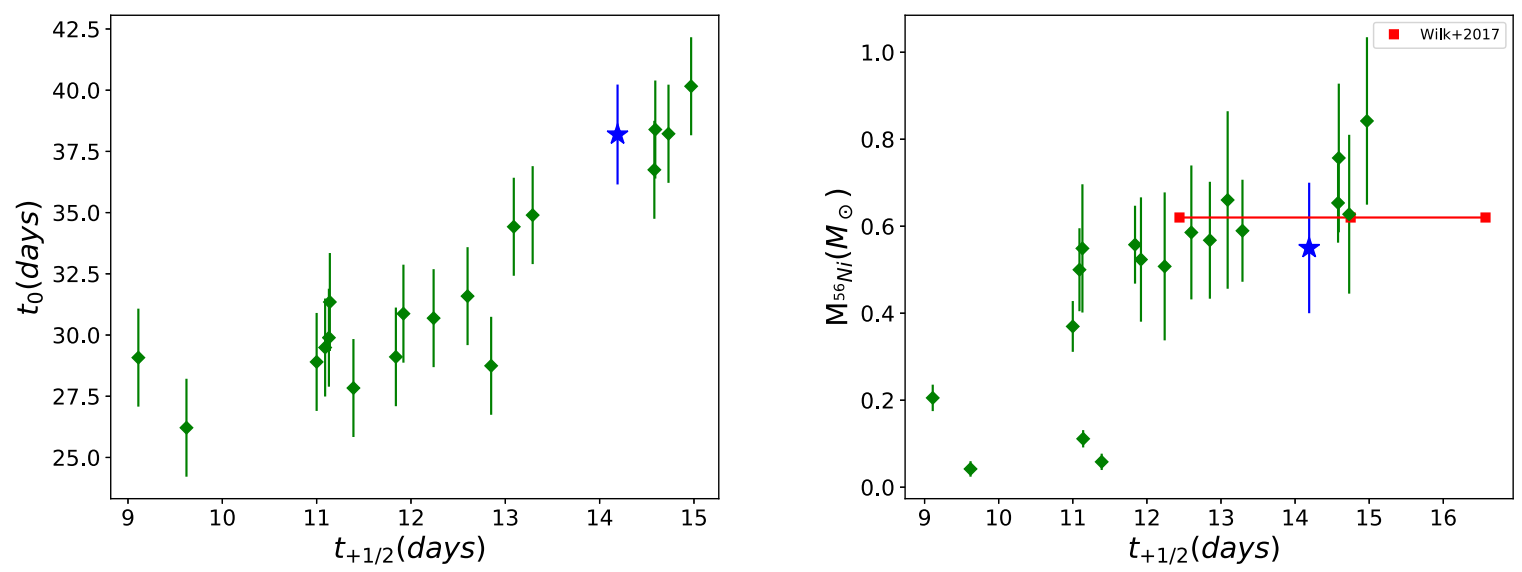

Figure 4. Left: $t_{0}$ is plotted against $t_{+1 / 2}$ for the sample of objects with sufficient late time data to measure $t_{0}$. There is a strong correlation between the two parameters, with $r \sim 0.85$. iPTF16abc is shown as the blue star and is consistent with the relation. Right: $\mathrm{M}_{56}{ }_{\mathrm{Ni}}$ is plotted against $t_{+1 / 2}$, the red points are predictions from the models of Wilk, Hillier \& Dessart (2017) for sub- $M_{\mathrm{Ch}}, M_{\mathrm{Ch}}$, and super- $M_{\mathrm{Ch}}$ progenitors.

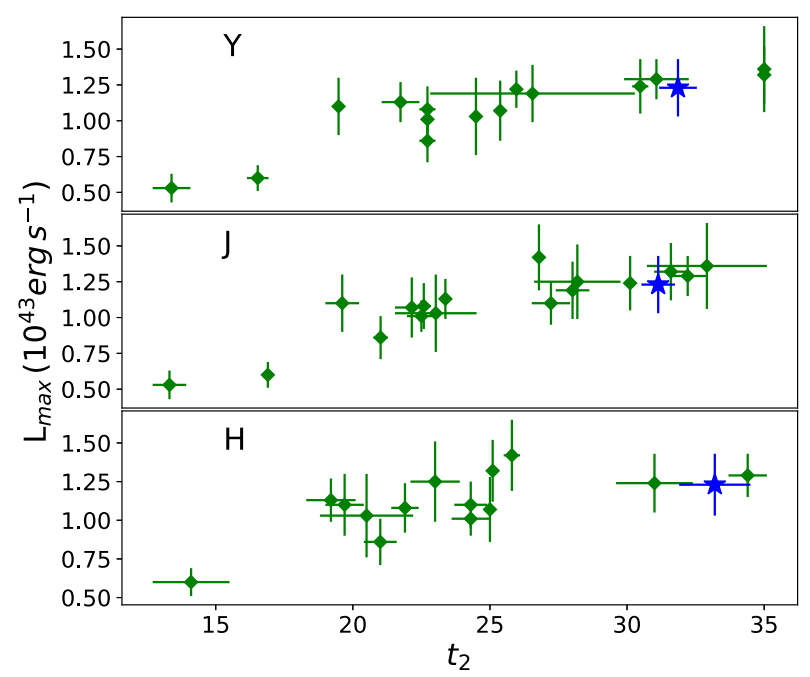

Figure 5. The (pseudo-) bolometric peak luminosity is plotted against the timing of the second maximum in the $Y J H$ filters. For both parameters, in this figure, the rise time is assumed to be $19 \mathrm{~d}$. The blue star is iPTF16abc. It lies on the relation for all other SNe Ia, though towards the brighter side.

$M_{\text {ej }}$ investigated in Wilk et al. (2017) is $1.02 \mathrm{M}_{\odot}$, lower $t_{+1 / 2}$ could imply smaller ejecta masses for the lower luminosity $\mathrm{SNe}$ in the sample (see also Blondin et al. 2017).

\subsection{Rise time}

In this section, we discuss the effect of using different prescriptions for the rise time as an input to calculate the $\mathrm{M}_{56 \mathrm{Ni}}$ and $t_{0}$ (see Tables 5 and 6). Although iPTF16abc has early-time observations that can strongly constrain the rise ( $\sim 18 \mathrm{~d}$ in the $g$ band, Miller et al. 2018), for a sample of SNe, the early behaviour is not as well characterized. Our canonical assumption in Section 3 was to use a rise time of $19 \mathrm{~d}$ along with an error of $3 \mathrm{~d}$ to capture the diversity in the observed values for the population of SNe Ia (e.g. Ganeshalingam, Li \& Filippenko 2011). In Ganeshalingam et al. (2011), the authors find that the rise time is correlated with the post-peak decline rate and they derive a linear relation between the $B$-band rise time and

$$
\begin{aligned}
& \Delta m_{15, \mathrm{~B}}: \\
& t_{\mathrm{R}, \mathrm{B}}=17.5-5 \cdot\left(\Delta m_{15, \mathrm{~B}}-1.1\right)
\end{aligned}
$$

The bolometric maximum occurs on average one day before $B_{\max }$ (Contardo et al. 2000; Scalzo et al. 2014), hence, we derive the rise time using

$t_{\mathrm{R}, \mathrm{bol}}=16.5-5 \cdot\left(\Delta m_{15, \mathrm{~B}}-1.1\right)$

Hence, faster declining SNe Ia would have a faster rise. From equation (1), we can see that a faster rise time implies a smaller $\mathrm{M}_{56 \mathrm{Ni}}$ for the same $L_{\max }$. The error on the rise from this method is $\sim 2 \mathrm{~d}$ (Scalzo et al. 2014). The impact of the rise time on the inferred $\mathrm{M}_{56 \mathrm{Ni}}$ is shown in Table 5. It is clear from the table that even with a varying rise time across the sample, the $\mathrm{M}_{56}{ }_{\mathrm{Ni}}$ estimates are within the errors.

We note that the estimation of $t_{0}$ from the (pseudo)-bolometric light curve, depends on the inference of the number of ${ }^{56}$ Niatoms, which in turn is sensitive to the $\alpha$ parameter. Since the deviations from the $\alpha=1$ case are of the order 10-15 per cent, the difference in the estimated $t_{0}$ is within the errors.

\subsection{Comparison with explosion models}

We computed $t_{0}$ and $\mathrm{M}_{56 \mathrm{Ni}}$ for a sample of $\mathrm{SNe}$ and found that these quantities were weakly correlated (see also Stritzinger et al. 2006; Scalzo et al. 2014). iPTF16abc lies on the $t_{0}-\mathrm{M}_{56 \mathrm{Ni}}$ relation for the SNe studied here, suggesting that the amount of $\mathrm{M}_{56 \mathrm{Ni}}$ and the progenitor mass for the $\mathrm{SNe}$ is compatible with the normal population of SNe Ia. Here, we compare the relation between $t_{0}$ and $L_{\max }$ to predictions from different theoretical models, since we want to directly compare observable quantities from the data to the model predictions. A range of $t_{0}$ values from $\sim 25$ to $40 \mathrm{~d}$ in itself would suggest that not all $\mathrm{SNe}$ Ia could arise from the same ejecta mass (Stritzinger et al. 2006; Scalzo et al. 2014).

In Fig. 8, we plot the $t_{0}$ against the $L_{\max }$ for the sample of $\mathrm{SNe}$ in this study and compare them to the predictions from various explosion scenarios. The synthetic observables were taken from the Heidelberg Supernova Model Archive (HESMA; Kromer, Ohlmann \& Roepke 2017). We find that the bright end of the distribution appears to be consistent with the $M_{\mathrm{ch}}$ delayed detonation models of Seitenzahl et al. (2013). The faint end of the relation is traced well by the sub- $M_{\text {ch }}$ models of Fink et al. (2010) and Sim et al. (2010) although 
Table 6. The derived $\mathrm{M}_{56} \mathrm{Ni}$ for the sample of SNe with a measured $t_{0}$ under two different assumptions on the rise time of the SNe. From the values below, we find that the inferred $\mathrm{M}_{56 \mathrm{Ni}}$ is within the errors even with individual rise times for each $\mathrm{SN}$.

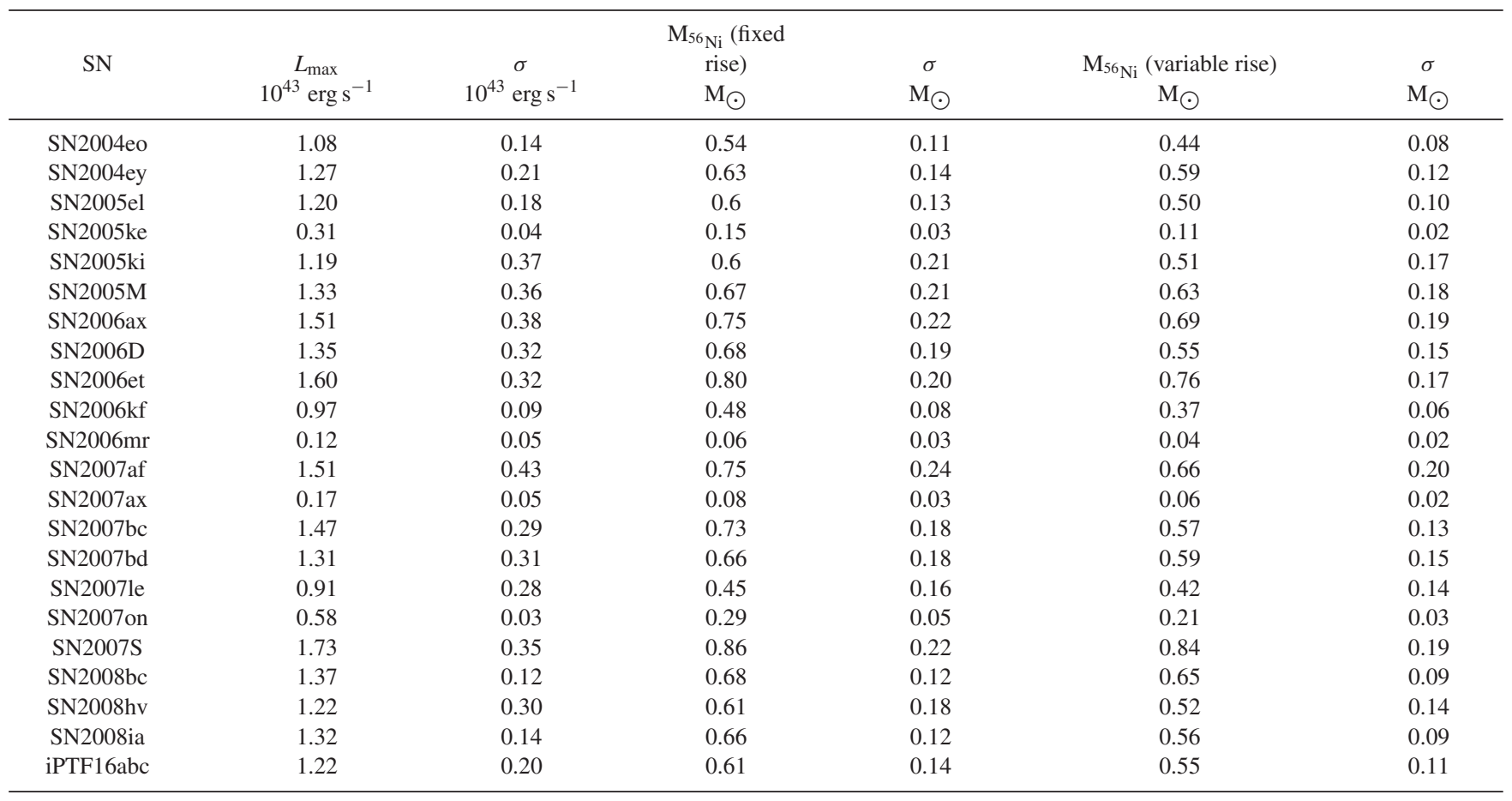

Table 7. The values of the transparency time-scales for a sample of SNe from the literature with sufficient data. The first estimate assume a rise time of $19 \mathrm{~d}$, whereas the second assumes a rise time that varies as a function of the measured $\Delta m_{15}(B)$. An additional systematic uncertainty of $\sim 2 \mathrm{~d}$ should be added in quadrature to the error on $t_{0}$ due to the uncertainty in knowing the rise time. The values from the two different estimates are comparable within the systematic errors..

\begin{tabular}{|c|c|c|c|c|}
\hline SN & $\begin{array}{c}t_{0}(\text { fix rise }) \\
\text { (d) }\end{array}$ & $\begin{array}{l}\text { err } \\
\text { (d) }\end{array}$ & $\begin{array}{c}t_{0}(\text { variable rise }) \\
\text { (d) }\end{array}$ & $\begin{array}{l}\text { err } \\
\text { (d) }\end{array}$ \\
\hline SN2004eo & 35.34 & 0.06 & 36.67 & 0.08 \\
\hline SN2005el & 28.38 & 0.03 & 29.49 & 0.05 \\
\hline SN2005ke & 28.87 & 0.02 & 31.35 & 0.05 \\
\hline SN2005ki & 29.85 & 0.04 & 30.69 & 0.06 \\
\hline SN2006D & 28.47 & 0.02 & 29.89 & 0.05 \\
\hline SN2006et & 38.18 & 0.09 & 38.39 & 0.09 \\
\hline SN2006kf & 27.62 & 0.04 & 28.90 & 0.06 \\
\hline SN2006mr & 24.54 & 0.01 & 26.21 & 0.02 \\
\hline SN2007af & 33.50 & 0.06 & 34.42 & 0.08 \\
\hline SN2007ax & 26.12 & 0.02 & 27.84 & 0.04 \\
\hline SN2008bc & 36.60 & 0.05 & 36.75 & 0.05 \\
\hline SN2008hv & 29.88 & 0.04 & 30.87 & 0.07 \\
\hline SN2008ia & 28.42 & 0.22 & 29.11 & 0.24 \\
\hline iPTF16abc & 39.50 & 0.21 & 39.76 & 0.22 \\
\hline
\end{tabular}

some of the predictions from the most massive sub- $M_{\mathrm{ch}}$ models seem consistent with the brightest observed $\mathrm{SNe}$ too. The violent merger models imply a longer transparency time-scale than observed for any $\mathrm{SNe}$ in the sample. iPTF16abc is consistent with the observed relation and appears to be closer to the trend for the $M_{\mathrm{ch}}$ models.
We find our conclusion that sub- $M_{\text {ch }}$ scenario are consistent with nearly the entire range of observed $L_{\max }$ and $t_{0}$, whereas the $M_{\mathrm{ch}}$ can only explain the bright end is supported by recent theoretical studies which find that the entire width-luminosity relation reproducable by sub-Chandra models, whereas the Chandrasekhar mass mod- 
Table 8. The values of the (pseudo-) bolometric peak luminosities and the transparency time-scales for a sample of SNe from the literature with sufficient data to measure both quantities. An additional systematic uncertainty of $\sim 2 \mathrm{~d}$ should be added in quadrature to the error on $t_{0}$ due to the uncertainty in knowing the rise time.

\begin{tabular}{cccc}
\hline SN & $\begin{array}{c}\mathrm{t}_{+1 / 2} \\
(\mathrm{~d})\end{array}$ & $\begin{array}{c}t_{0}(\text { variable rise }) \\
(\mathrm{d})\end{array}$ & $\begin{array}{c}\text { err } \\
(\mathrm{d})\end{array}$ \\
\hline SN2004ey & 13.29 & 34.9 & 0.03 \\
SN2005el & 11.09 & 29.49 & 0.05 \\
SN2005ke & 11.14 & 31.35 & 0.05 \\
SN2005ki & 12.24 & 30.69 & 0.06 \\
SN2005M & 14.73 & 38.22 & 0.14 \\
SN2006D & 11.13 & 29.89 & 0.05 \\
SN2006et & 14.59 & 38.39 & 0.09 \\
SN2006kf & 11.00 & 28.9 & 0.06 \\
SN2006mr & 9.62 & 26.21 & 0.02 \\
SN2007af & 13.09 & 34.42 & 0.08 \\
SN2007ax & 11.39 & 27.84 & 0.04 \\
SN2007bc & 12.85 & 28.74 & 31.59 \\
SN2007bd & 12.60 & 29.08 & 0.05 \\
SN2007on & 9.11 & 40.16 & 0.01 \\
SN2007S & 14.97 & 36.75 & 0.06 \\
SN2008bc & 14.58 & 30.87 & 0.09 \\
SN2008hv & 11.92 & 29.11 & 0.05 \\
SN2008ia & 11.84 & 39.76 & 0.07 \\
iPTF16abc & 14.14 & 0.24 & 0.09 \\
\hline
\end{tabular}

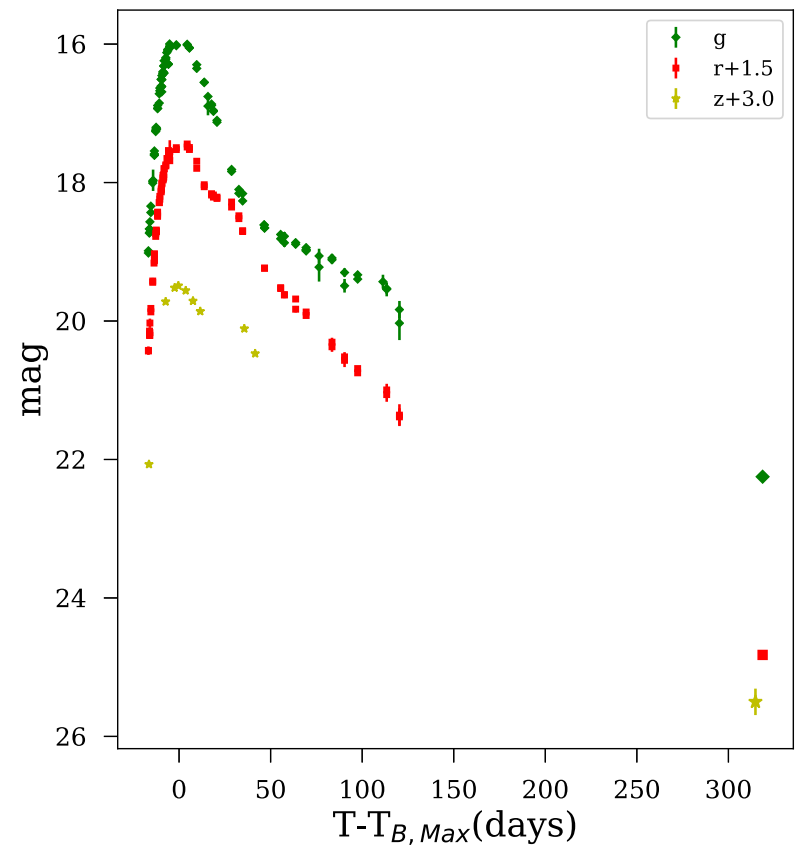

Figure 6. $g, r, z$ light curves of iPTF16abc from Miller et al. (2018), along with the nebular phase $(>+300 \mathrm{~d})$ data points from the DECaLS survey. The legend gives the magnitude offsets for the $r$ and $z$ light curves.

els only reproduce the bright end (Goldstein \& Kasen 2018). This conclusion is also supported by a comparison of observations and model predictions presented in Shen et al. (2017). The two overluminous SNe in our sample with SNID classifications of 91T-like (Folatelli et al. 2013) are both consistent with $M_{\mathrm{ch}}$ model prediction. Fisher \& Jumper (2015) suggest that $M_{\text {ch }}$ WD explosions that lack a vigourous deflagration phase would produce $91 \mathrm{~T}$-like SNe. Since these explosions would also have higher ${ }^{56}$ Nimixing, this might in-

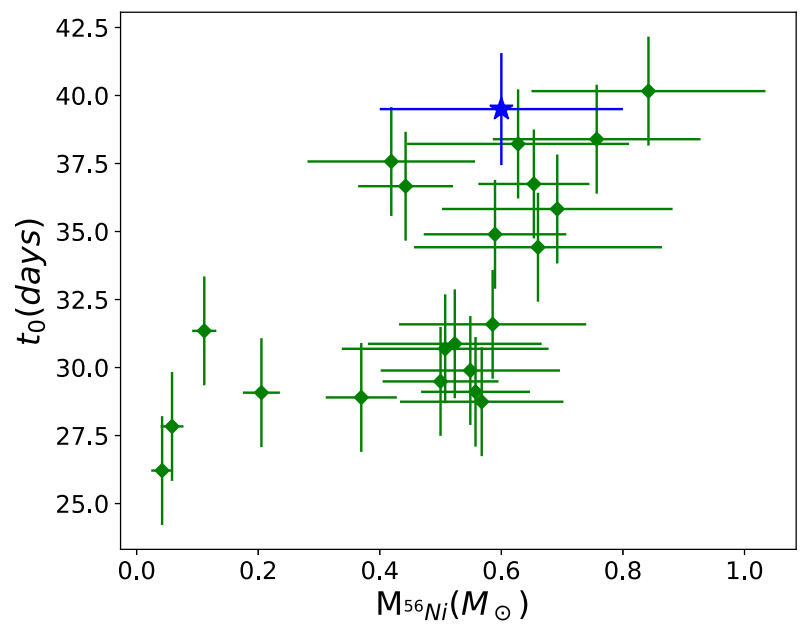

Figure 7. The transparency time-scale, $t_{0}$, is plotted against the ${ }^{56} \mathrm{Ni}$ mass for a sample of SN Ia with $u \rightarrow H$ band coverage (green diamonds). There is a weak correlation $(r \sim 0.67)$ between the two parameters. iPTF16abc is plotted as a blue star and appears to be consistent with the general trend of $t_{0}$ versus $M_{56 \mathrm{Ni}}$.

dicate that overluminous $\mathrm{SNe}$ that show 91T-like spectral features have shorter deflagration phases.

We note that iPTF16abc appears to have $t_{0}$ estimates consistent with $M_{\mathrm{ej}}=1.4 \mathrm{M}_{\odot}$, under the assumption that $q \sim 1 / 3$. This value of $q$ would point towards strong ${ }^{56} \mathrm{Nimixing}$ in the ejecta in line with the findings of Miller et al. (2018). Miller et al. (2018) cite a discrepancy between the early-time observables and the early colours predicted for the sub-Chandrasekhar 'double detonations' of Noebauer et al. (2017) as a reason that iPTF16abc is likely not a result of the double detonation of a sub- $M_{\mathrm{ch}} \mathrm{WD}$. The late-time bolometric properties would point to a similar conclusion.

Using the transparency time-scale, we can calculate the epoch at which the energy deposition from the $\gamma$-rays is equal to the 


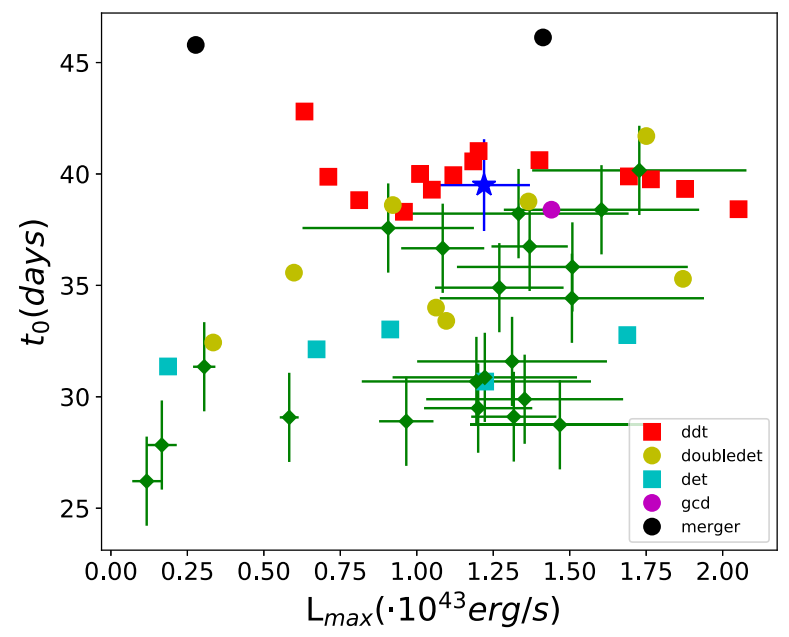

Figure 8. The transparency time-scale is plotted against the peak bolometric luminosity for the sample of SNe in this study. Overplotted are the expectations from the different explosion scenarios. The red squares are the three-dimensional $M_{\mathrm{ch}}$ delayed detonation models of Seitenzahl et al. (2013), the magenta circle is the $M_{\mathrm{ch}}$ gravitationally confined detonation of Seitenzahl et al. (2016). The yellow circles are the sub- $M_{\mathrm{ch}}$ 'doubledetonation' models of Fink et al. (2010), whereas the yellow squares are the sub- $M_{\text {ch }}$ pure detonations presented in Sim et al. (2010). The two black circles are the violent merger models of Pakmor et al. (2010, 2012).

contribution from the positrons (hereafter, $t_{\mathrm{c}}$, see Childress et al. 2015). For iPTF16abc, $t_{\mathrm{c}}=205.2 \pm 11.3 \mathrm{~d}$ which is consistent with the calculated value for the $M_{\mathrm{ch}}$ delayed detonation model (DDC10) of Blondin et al. (2013) of $t_{\mathrm{c}} \sim 214 \mathrm{~d}$, compared to values between 160 and $180 \mathrm{~d}$ for sub- $M_{\text {ch }}$ models (Sim et al. 2010; Fink et al. 2010). This direct comparison adds further evidence for iPTF16abc being the result of $M_{\mathrm{ch}}$ explosion.

\subsection{Comparison to overluminous SNe Ia}

In Fig. 8, we see that brighter objects have longer transparency time-scales and these objects are more consistent with the predictions from Chandrasekhar mass models than sub-Chandra models. Some of these high-luminosity SNe are classified as 91T-like (e.g. SN2005M, SN2007S, Folatelli et al. 2013), whereas others are classified as normal (e.g. SN2006ax, SN2008bc, Folatelli et al. 2013). Hence, this would suggest that overluminous SN Ia have similar maximum and post-maximum bolometric properties and possibly similar progenitor masses, independent of the early time spectroscopic differences (e.g. Miller et al. 2018; Contreras et al. 2018). Overluminous SNe with early observations also show a linear rise ( $\sim 3 \mathrm{~d}$ after first light) which could be signs of strong mixing of ${ }^{56} \mathrm{Ni}$ (Magee et al. 2018). Hence, the extent of ${ }^{56}$ Nimixing could be a parameter governing the early time photometric and spectroscopic diversity of SNe Ia. These distinct spectroscopic features are also seen in $M_{\text {ch }}$ models which lack a vigorous deflagration phase (Fisher \& Jumper 2015), further adding to the evidence that the progenitors of overluminous SNe Ia could be Chandrasekhar mass.

The width of the [Fe III $] 4700 \AA$ line in the nebular phase spectrum for iPTF16abc is consistent with the values for normal SNe Ia, but towards the higher end of the distribution. This might indicate that the extent of the IGE core is higher than average for SNe Ia, possible further evidence for strong mixing in the ejecta. It is, however, hard to draw strong conclusions since the nebular spectra in the optical consist of several line blends.

\section{CONCLUSIONS}

iPTF16abc has an excellent set of early-time observations that showed a peculiar rise time and unusual spectroscopic features (Miller et al. 2018). In this study, we present a nebular spectrum of the SN and nebular phase photometry in the optical. We analyse the early- and late-time observations of iPTF16abc in context of a sample of SNe Ia from the literature.

We measure the bolometric peak luminosity, ${ }^{56} \mathrm{Nimasses}$ and transparency time-scales for $21 \mathrm{SNe}$ and find a weak correlation between $t_{0}$ and ${ }^{56} \mathrm{Nimass}$ indicating that $\mathrm{SNe}$ that produce more ${ }^{56}$ Nialso become transparent at a later epoch (see also Stritzinger et al. 2006; Scalzo et al. 2014). iPTF16abc lies on the relation between $t_{0}$ and $\mathrm{M}_{56 \mathrm{Ni}}$, although it is on the brighter end of the distribution. Additionally, we measure $t_{+1 / 2}$ (Table 7), the bolometric time of half-light and compare the inferred values to the predictions from models of Wilk et al. (2017). The observed range of $t_{+1 / 2}$ values is larger than the range from the models which have ejecta mass values between 1.02 and $1.70 \mathrm{M}_{\odot}$, implying a large range of ejecta masses for the SNe.

The transitional and nebular spectrum of iPTF16abc appear qualitatively similar to the normal SN $2011 \mathrm{fe}$ as well as to overluminous SNe 1991T and 1999aa. The absolute magnitude of iPTF16abc in the nebular phase is consistent with normal SNe from the literature. iPTF16abc is also consistent with the relation between the FWHM of the $4700 \AA$ feature and $\Delta m_{15}(B)$ for SNe from the literature (Blondin et al. 2012; Silverman et al. 2013), though it lies towards the high line width end of the relation. This would suggest that the peculiarities seen in the early time spectra of iPTF16abc are not present at late epochs, hence, arguing for obtaining data at both early and late epochs to understand the explosion properties of $\mathrm{SNe}$ Ia.

From Fig. 8, we find that the sub- $M_{\mathrm{ch}}$ models appear to explain a large fraction of the $t_{0}-L_{\max }$ relation, though the bright end is still better explained by the $M_{\mathrm{ch}}$ delayed detonation. This could be indicative of multiple explosion mechanisms explaining the observed diversity of SNe Ia. iPTF16abc appears to be consistent with the properties of $M_{\mathrm{ch}}$ explosion scenario.

\section{ACKNOWLEDGEMENTS}

We would like to thank Peter Nugent for pointing us to the DECaLS photometry of iPTF16abc. We acknowledge fruitful comments from Jesper Sollerman. Funding from the Swedish Research Council, the Swedish Space Board, and the Knut \& Alice (K\&A) Wallenberg foundation made this research possible. A.A.M. is funded by the Large Synoptic Survey Telescope Corporation in support of the Data Science Fellowship Program. This work made use of the Heidelberg Supernova Model Archive (HESMA), https://hesma.h-its.org.

\section{REFERENCES}

Amanullah R., Goobar A., 2011, ApJ, 735, 20

Arnett W. D., 1982, ApJ, 253, 785

Ashall C. et al., 2018, MNRAS, 477, 153

Biscardi I. et al., 2012, A\&A, 537, A57

Blondin S. et al., 2012, AJ, 143, 126

Blondin S., Dessart L., Hillier D. J., Khokhlov A. M., 2013, MNRAS, 429, 2127

Blondin S., Dessart L., Hillier D. J., 2015, MNRAS, 448, 2766

Blondin S., Dessart L., Hillier D. J., Khokhlov A. M., 2017, MNRAS, 470, 157

Bloom J. S. et al., 2012, ApJ, 744, L17 
Bulla M., Goobar A., Amanullah R., Feindt U., Ferretti R., 2018, MNRAS, 473, 1918

Burns C. R. et al., 2011, AJ, 141, 19

Burns C. R. et al., 2014, ApJ, 789, 32

Cao Y. et al., 2015, Nature, 521, 328

Cardelli J. A., Clayton G. C., Mathis J. S., 1989, ApJ, 345, 245

Childress M. J. et al., 2015, MNRAS, 454, 3816

Contardo G., Leibundgut B., Vacca W. D., 2000, A\&A, 359, 876

Contreras C. et al., 2010, AJ, 139, 519

Contreras C. et al., 2018, ApJ, 859, 24

Dhawan S., Leibundgut B., Spyromilio J., Maguire K., 2015, MNRAS, 448, 1345

Dhawan S., Leibundgut B., Spyromilio J., Blondin S., 2016, A\&A, 588, A84

Dhawan S., Leibundgut B., Spyromilio J., Blondin S., 2017, A\&A, 602, A118

Elias J. H., Frogel J. A., Hackwell J. A., Persson E. E., 1981, ApJ, 251, L13

Elias J. H., Matthews K., Neugebauer G., Persson S. E., 1985, ApJ, 296, 379

Ferretti R. et al., 2017, A\&A, 606, A111

Filippenko A. V. et al., 1992, ApJ, 384, L15

Fink M., Röpke F. K., Hillebrandt W., Seitenzahl I. R., Sim S. A., Kromer M., 2010, A\&A, 514, A53

Fisher R., Jumper K., 2015, ApJ, 805, 150

Folatelli G., Morrell N., Phillips M. M. et al., 2013, ApJ, 773, 53

Fransson C., Jerkstrand A., 2015, ApJ, 814, L2

Friedman A. S. et al., 2015, ApJS, 220, 9

Ganeshalingam M., Li W., Filippenko A. V., 2011, MNRAS, 416, 2607

Garavini G. et al., 2004, AJ, 128, 387

Goldstein D. A., Kasen D., 2018, ApJ, 852, L33

Gómez G., López R., 1998, AJ, 115, 1096

Goobar A. et al., 2014, ApJ, 784, L12

Goobar A. et al., 2015, ApJ, 799, 106

Graham M. L. et al., 2017, MNRAS, 472, 3437

Hillebrandt W., Kromer M., Röpke F. K., Ruiter A. J., 2013, Front. Phys., 8,116

Hosseinzadeh G. et al., 2017, ApJ, 845, L11

Hoyle F., Fowler W. A., 1960, ApJ, 132, 565

Jeffery D. J., 1999, preprint ( astro-ph/9907015)

Jiang J.-A., Doi M., Maeda K. et al., 2017, Nature, 550, 80

Kasen D., 2006, ApJ, 649, 939

Kasen D., 2010, ApJ, 708, 1025

Kromer M. et al., 2013, MNRAS, 429, 2287

Kromer M. et al., 2016, MNRAS, 459, 4428

Kromer M., Ohlmann S. T., Roepke F. K., 2017, Mem. Soc. Astron. Ital., 88,312

Lair J. C., Leising M. D., Milne P. A., Williams G. G., 2006, AJ, 132, 2024

Leibundgut B., 1988, PhD thesis

Maeda K. et al., 2011, MNRAS, 413, 3075

Magee M. R., Sim S. A., Kotak R., Kerzendorf W. E., 2018, A\&A, 614, A115

Maguire K. et al., 2014, MNRAS, 444, 3258

Maguire K., Taubenberger S., Sullivan M., Mazzali P. A., 2016, MNRAS, 457, 3254

Mandel K. S., Wood-Vasey W. M., Friedman A. S., Kirshner R. P., 2009, ApJ, 704, 629
Maoz D., Mannucci F., Nelemans G., 2014, ARA\&A, 52, 107

Marion G. H. et al., 2016, ApJ, 820, 92

Matheson T. et al., 2008, AJ, 135, 1598

Mazzali P. A., Danziger I. J., Turatto M., 1995, A\&A, 297, 509

Mazzali P. A., Chugai N., Turatto M., Lucy L. B., Danziger I. J., Cappellaro E., della Valle M., Benetti S., 1997, MNRAS, 284, 151

Mazzali P. A., Cappellaro E., Danziger I. J., Turatto M., Benetti S., 1998, ApJ, 499, L49

Mazzali P. A. et al., 2014, MNRAS, 439, 1959

Miller A. A. et al., 2018, ApJ, 852, 100

Noebauer U. M., Kromer M., Taubenberger S., Baklanov P., Blinnikov S., Sorokina E., Hillebrandt W., 2017, MNRAS, 472, 2787

Nugent P. E. et al., 2011, Nature, 480, 344

Pakmor R., Kromer M., Röpke F. K., Sim S. A., Ruiter A. J., Hillebrandt W., 2010, Nature, 463, 61

Pakmor R., Kromer M., Taubenberger S., Kromer M., Taubenberger S., Sim S. A., Röpke F. K., Hillebrandt W., 2012, ApJ, 747, L10

Pereira R. et al., 2013, A\&A, 554, A27

Phillips M. M., Wells L. A., Suntzeff N. B., Hamuy M., Leibundgut B., Kirshner R. P., Foltz C. B., 1992, AJ, 103, 1632

Ruiz-Lapuente P., Cappellaro E., Turatto M., Gouiffes C., Danziger I. J., della Valle M., Lucy L. B., 1992, ApJ, 387, L33

Sahu D. K. et al., 2008, ApJ, 680, 580

Sasdelli M., Mazzali P. A., Pian E., Nomoto K., Hachinger S., Cappellaro E., Benetti S., 2014, MNRAS, 445, 711

Scalzo R. et al., 2014, MNRAS, 440, 1498

Schlafly E. F., Finkbeiner D. P., 2011, ApJ, 737, 103

Seitenzahl I. R. et al., 2013, MNRAS, 429, 1156

Seitenzahl I. R. et al., 2016, A\&A, 592, A57

Shen K. J., Kasen D., Miles B. J., Townsley D. M., 2017, ApJ, 854, 52

Silverman J. M., Ganeshalingam M., Filippenko A. V., 2013, MNRAS, 430, 1030

Sim S. A., Röpke F. K., Hillebrandt W., Kromer M., Pakmor R., Fink M., Ruiter A. J., Seitenzahl I. R., 2010, ApJ, 714, L52

Stritzinger M., Sollerman J., 2007, A\&A, 470, L1

Stritzinger M., Leibundgut B., Walch S., Contardo G., 2006, A\&A, 450, 241

Stritzinger M. D. et al., 2011, AJ, 142, 156

Swartz D. A., Sutherland P. G., Harkness R. P., 1995, ApJ, 446, 766

Taubenberger S. et al., 2015, MNRAS, 448, L48

Wilk K., Hillier D. J., Dessart L. MNRAS, 474, 3187

Zhang J.-J. et al., 2016, ApJ, 817, 114

Zheng W. et al., 2013, ApJ, 778, L15

Zheng W. et al., 2014, ApJ, 783, L24

\section{APPENDIX A: RADIOACTIVE DECAY ENERGY FITS TO BOLOMETRIC LIGHT CURVES}

In Section 3.2, we describe the procedure for fitting a radioactive decay energy deposition function to the bolometric light curve. Here, we present the light-curve fits for the $\mathrm{SNe}$ in our sample. We plot the deposition curve for the best-fitting value of the transparency time-scale and along with it the curve for complete trapping and complete escape. 

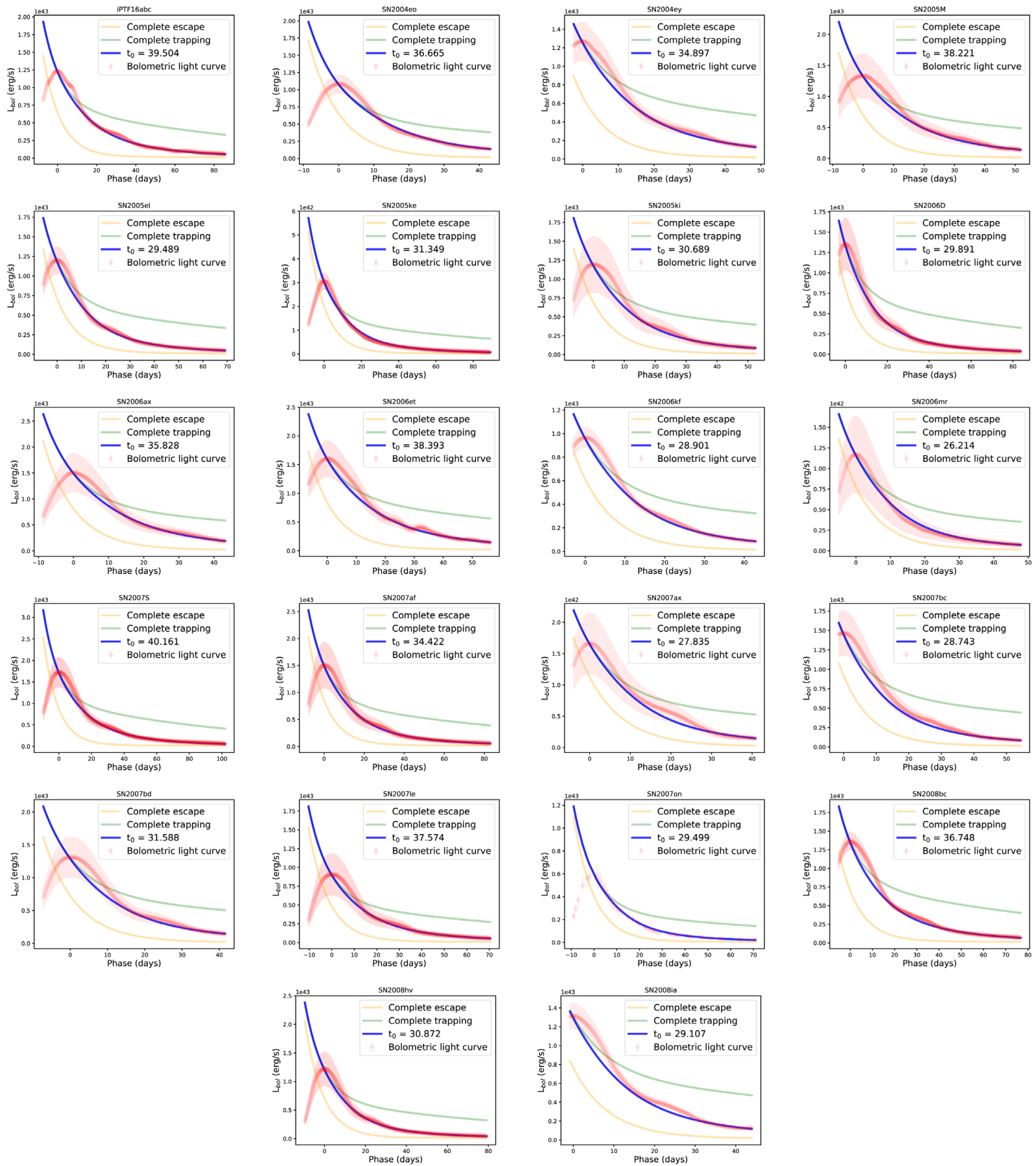

Figure A1. The radioactive decay energy deposition fits to the bolometric light curve for the sample of SNe analysed in this study. For each SN, the fits are presented along with the constructed light curve and the deposition function for complete trapping and complete escape of $\gamma$-rays.

This paper has been typeset from a $\mathrm{T}_{\mathrm{E}} \mathrm{X} / \mathrm{L} \mathrm{T} \mathrm{EX}$ file prepared by the author. 